\title{
Alu insertion polymorphisms shared by Papio baboons and Theropithecus gelada reveal an intertwined common ancestry
}

\author{
Jerilyn A. Walker ${ }^{1 \dagger}$, Vallmer E. Jordan ${ }^{1 \dagger}$, Jessica M. Storer ${ }^{1}$, Cody J. Steely ${ }^{1}$, Paulina Gonzalez-Quiroga', \\ Thomas O. Beckstrom ${ }^{1}$, Lydia C. Rewerts', Corey P. St. Romain ${ }^{1}$, Catherine E. Rockwell ${ }^{1}$, Jeffrey Rogers ${ }^{2,3}$, \\ Clifford J. Jolly ${ }^{4}$, Miriam K. Konkel ${ }^{1,5}$, The Baboon Genome Analysis Consortium and Mark A. Batzer ${ }^{1 *}$ (D)
}

\begin{abstract}
Background: Baboons (genus Papio) and geladas (Theropithecus gelada) are now generally recognized as close phylogenetic relatives, though morphologically quite distinct and generally classified in separate genera. Primate specific Alu retrotransposons are well-established genomic markers for the study of phylogenetic and population genetic relationships. We previously reported a computational reconstruction of Papio phylogeny using large-scale whole genome sequence (WGS) analysis of Alu insertion polymorphisms. Recently, high coverage WGS was generated for Theropithecus gelada. The objective of this study was to apply the high-throughput "poly-Detect" method to computationally determine the number of Alu insertion polymorphisms shared by T. gelada and Papio, and vice versa, by each individual Papio species and T. gelada. Secondly, we performed locus-specific polymerase chain reaction (PCR) assays on a diverse DNA panel to complement the computational data.

Results: We identified 27,700 Alu insertions from T. gelada WGS that were also present among six Papio species, with nearly half $(12,956)$ remaining unfixed among 12 Papio individuals. Similarly, each of the six Papio species had species-indicative Alu insertions that were also present in T. gelada. In general, P. kindae shared more insertion polymorphisms with T. gelada than did any of the other five Papio species. PCR-based genotype data provided additional support for the computational findings.

Conclusions: Our discovery that several thousand Alu insertion polymorphisms are shared by T. gelada and Papio baboons suggests a much more permeable reproductive barrier between the two genera then previously suspected. Their intertwined evolution likely involves a long history of admixture, gene flow and incomplete lineage sorting.
\end{abstract}

Keywords: Retrotransposon, Evolutionary biology, Primate phylogeny, Alu element

\section{Background}

The phylogenetic position of the gelada (Theropithecus gelada) has been debated since the species was first scientifically described in 1835 by Rüppell. Originally named Macacus gelada, it was later placed in a genus of its own by I. Geoffroy Saint-Hilaire (1843) [1] where it remains today as the only extant species of Theropithecus [2]. By contrast, there are currently six recognized species of Papio baboons distributed across most of sub-Saharan

\footnotetext{
* Correspondence: mbatzer@lsu.edu

† Jerilyn A. Walker and Vallmer E. Jordan contributed equally to this work.

${ }^{1}$ Department of Biological Sciences, Louisiana State University, 202 Life

Sciences Building, Baton Rouge, Louisiana 70803, USA

Full list of author information is available at the end of the article
}

Africa [3-5]. Evidence from morphological comparisons and mitochondrial and whole genome sequencing (WGS) all support a primary phylogenetic division into northern ( $P$. anubis, $P$. papio and $P$. hamadryas) and southern ( $P$. ursinus, $P$. cynocephalus and $P$. kindae) clades [5-7]. The genetics of the baboon species complex have been studied much more extensively [4-14] than that of the mountain dwelling geladas of the Ethiopian highlands [15-20]. Theropithecus is estimated to have diverged from a Papio-like ancestor about 4-5 million years ago (mya) based on fossil evidence $[2,4,21,22]$ and analysis of mitochondrial DNA [23], while extant Papio species began to diversify about 2 mya $[5,7,9,24]$. 
A complex history of evolution has been reported among extant species within the genus Papio [5-7, 25-28], in which mitochondrial and phenotypically-based phylogenies of the six currently recognized extant species frequently conflict. Recently, the Baboon Genome Analysis Consortium published a study of the complex population history of Papio baboons based on whole genome sequences, providing evidence for multiple episodes of introgression and admixture throughout radiation of the genus and a long history of genetic exchange among diverging lineages that were presumably phenotypically distinct [6].

Primate specific Alu retrotransposons are wellestablished genomic markers for the study of population genetic and phylogenetic relationships [27, 29-40]. Alu element insertions are considered unique events, have a known directionality where the ancestral state is known to be the absence of the element, and are relatively inexpensive to genotype [33, 41-45]. Alu insertions shared by individuals or species are widely accepted as largely being inherited from a common ancestor. The amplification of $A l u$ elements has been ongoing in primate genomes since the origin of the Order, about 65 mya [42, 46, 47]. Alu elements mobilize via a "copy and paste" mechanism through an RNA intermediate, a process termed "target-primed reverse transcription" (TPRT) [48]. We recently reported a computational reconstruction of Papio phylogeny using 187,000 Alu insertions identified through a large-scale whole genome sequence analysis [26]. This study not only determined the most likely branching order within Papio with high statistical support, but also quantified the number of $A l u$ insertions supporting alternative topologies, demonstrating the efficacy of whole genome computational analysis of $A l u$ polymorphisms to identify and investigate complexities in phylogenetic relationships.

During the early stages of the Baboon Genome Analysis Consortium [6] an analysis of the [Panu_2.0] genome of Papio anubis revealed an occasional Alu element insertion that appeared to be present in T. gelada DNA based on PCR, while also remaining polymorphic among the six Papio species. Although intriguing given the estimated 45 mya divergence between the two genera, with no other WGS data available at the time for further computational screening, these insertions were set aside as being uninformative for resolving phylogenetic relationships within Papio. Recently, we have generated high coverage WGS data for an individual Theropithecus gelada (Sample name 36168, BioProject PRJNA251424, submitted by Baylor College of Medicine). Therefore, the objective of this study was to apply the "polyDetect" method [26] to computationally determine the number of $A l u$ insertion polymorphisms shared by the representative $T$. gelada genome and 12 individuals representing the genus Papio. Our approach targeted recently integrated Alu insertions present in T. gelada yet polymorphic within Papio and absent from rhesus macaque [Mmul8.0.1]. Alu insertions recent enough to remain polymorphic among Papio species would be expected to have integrated after the split from Theropithecus and therefore be absent from Theropithecus. Similarly, Theropithecus, with a much smaller effective population size [20], would be expected to have its own set of lineage-specific insertions. Observations of a large number of $A l u$ insertions present in both genera that remain unfixed in all species would suggest a long history of ancient admixture, extensive incomplete lineage sorting, or on-going hybridization [44]. Here, we have computationally ascertained a dataset of $A l u$ insertions present in the Theropithecus gelada WGS data that also remained polymorphic among 12 Papio baboons representing all six species. This analysis prompted a reciprocal computational comparison of WGS of each Papio individual to determine the number of $A l u$ insertion polymorphisms shared exclusively between each Papio species and $T$. gelada.

Locus specific PCR analyses were performed on a DNA panel which included samples from all six Papio species, T. gelada and rhesus macaque (Macaca mulatta) as an outgroup to provide experimental support for the computational findings.

\section{Methods \\ WGS samples}

Whole-genome sequencing was performed by the Baylor College of Medicine Human Genome Sequencing Center. All samples were sequenced to an average coverage of $32.4 x$ and minimum of $26.3 x$ [6]. The same dataset described in Jordan et al. (2018) [26] for 12 Papio individuals was used in this analysis along with WGS from a representative $T$. gelada genome. These samples are listed in Additional file 1. We used two individuals from each of the six extant Papio species (we randomly selected two individuals from $P$. anubis and $P$. kindae) to conduct our computational analysis; along with WGS data from the rhesus macaque sample used to build the recent $M$. mulatta assembly [Mmul8.0.1] and WGS data for one Theropithecus gelada (isolate 891096; sample name 38168; adult female captive born at the Bronx Zoo; NCBI BioProject PRJNA251424; Accession: SAMN06167567). WGS data were accessed from the NCBI-SRA database as described previously [26].

\section{Computational Alu detection}

We used the "polyDetect" computational pipeline [26] to perform our analysis. Our approach targeted recently integrated $A l u$ insertions present in $T$. gelada yet polymorphic within Papio and absent from rhesus macaque [Mmul8.0.1]. The approximate chromosomal position of 
each candidate insertion was estimated using a split-read method as described previously [26]. Briefly, for the alignment phase, we used BWA-MEM version 0.7.17r1188 [49] to map the sequencing reads to a consensus AluY sequence obtained from Repbase [50]. The Alu portion of each candidate split-read was cleaved allowing the remaining unique flanking sequence to be aligned to the rhesus macaque genome assembly [Mmul8.0.1] using bowtie2 version2.3.2 [51]. Split-reads were categorized as sequences that mapped uniquely to the AluY consensus sequence and the [Mmul8.0.1] assembly. The resulting genotypes, generated for all individuals in our panel, isolated thousands of phylogenetically informative markers. Data for these loci were sorted by the number of $A l u$ insertions common to T. gelada and any two to twelve Papio individuals. For purposes of the present analyses, those present in all 12 Papio individuals were considered fixed present in the dataset and eliminated from this portion of the study. For the reciprocal comparison, the Alu insertions detected in both individuals of a single Papio species, as reported previously in Jordan et al. (2018) [26], were sorted by their [Mmul8.0.1] predicted insertion coordinates and crossreferenced with coordinates from the $T$. gelada WGS reads to identify candidate shared insertion polymorphisms. These are listed in Additional file 1, Worksheet "Papio-Theropithecus."

\section{Statistical analysis of Alu insertion polymorphisms}

$A l u$ insertions predicted to be shared by $T$. gelada and any two to eleven of the twelve Papio individuals were considered polymorphic in the genus Papio and retained for further analysis. To determine if any particular species or clade had significantly different numbers of shared insertions with T. gelada, we performed a oneway analysis of variance (ANOVA) in Excel (alpha set at 0.05). A separate ANOVA was performed for each of the ten data bins representing two to eleven individuals. ANOVA "groups" were defined as either six Papio species with two individuals each, or two Papio clades (North / South) with six individuals each. If a significant 'between group' difference was detected, we followed with a Bonferroni post-hoc test in Excel, selecting the " $t$ Test: Two-sample assuming equal variances" function to perform a two-tailed t-test for $P \leq 0.05$. All $P$ values were recorded in Additional file 1: Table $\mathrm{S} 1$.

\section{Candidate Alu element selection and oligonucleotide primer design}

We randomly selected 150 candidate $A l u$ insertion polymorphisms from the first comparison (A: ascertained from the T. gelada WGS and polymorphic among Papio baboons) for in-house oligonucleotide primer design as described previously [52]. From the second comparison
(B: present in WGS of both individuals of a single Papio species and shared in T. gelada) we randomly selected about $10 \%$ of the candidate loci identified from each of the six Papio species, but no less than five loci from each species, for primer design. Oligonucleotide primers for PCR were designed using the predicted insertion coordinates from the rhesus macaque genome [Mmul8.0.1] since that was the "reference" genome used to map the T. gelada and Papio WGS reads. Suitable primer pairs were then analyzed against the Papio anubis baboon genome [Panu_2.0] using the "In-Silico PCR" tool in BLAT [53] through the University of California Santa Cruz (UCSC) Genome Browser [54]. If no PCR product was identified due to mismatches in the primer sequence, the primer pairs were analyzed by In-Silico PCR using the [Mmul8.0.1] assembly to obtain the predicted PCR product. This entire amplicon sequence was then analyzed using BLAT against the $P$. anubis genome [Panu_2.0] and checked for mismatches in order to design alternative oligonucleotide primers to help ensure PCR amplification in Papio baboons. Using this method we obtained estimates for our expected PCR product sizes in [Mmul8.0.1] and [Panu_2.0] (Additional file 2). Oligonucleotide primers for PCR were obtained from Sigma Aldrich (Woodlands, TX).

\section{Polymerase chain reaction assays}

The primate DNA panel used for PCR analyses was comprised of three $P$. anubis, one $P$. hamadryas, two $P$. papio, two $P$. cynocephalus, two $P$. ursinus, two $P$. kindae, one $T$. gelada, and a Macaca mulatta. A human (HeLa) sample was used as a positive control and TLE (10 mM Tris / 0.1 mM EDTA) was used as a negative control. Information about the samples is provided in Additional file 2 including their common name, origin, and ID.

A total of $172 \mathrm{Alu}$ insertion polymorphisms were retained in the dataset for PCR analyses. We used a subset of the computationally-derived Alu insertion polymorphisms ascertained from either A) T. gelada WGS and predicted to be shared in Papio, $(N=96)$; or B) Papio species WGS and predicted to be shared in T. gelada, $(N=52)$. We also included $N=24$ Alu loci previously ascertained from the reference genome of Papio anubis [Panu_2.0] (12 loci each from $[6,52])$ in which PCR results indicated the Alu insertion was present in T. gelada while remaining polymorphic among the six Papio species.

Oligonucleotide primers for PCR were designed using Primer3 software, either manually [55] for most of the Panu_2.0 derived candidate loci or using a modified version [56]. PCR amplifications were performed in $25 \mu \mathrm{l}$ reactions containing $25 \mathrm{ng}$ of template DNA; $200 \mathrm{nM}$ of each oligonucleotide primer; $1.5 \mathrm{mM} \mathrm{MgCl}_{2}, 10 \mathrm{x}$ PCR buffer (1x:50 mM KCl; 10 mM TrisHCl, pH 8.4); $0.2 \mathrm{mM}$ 
dNTPs; and 1-2 U Taq DNA polymerase. PCR reactions were performed under the following conditions: initial denaturation at $94{ }^{\circ} \mathrm{C}$ for $60 \mathrm{~s}$, followed by 32 cycles of denaturation at $94{ }^{\circ} \mathrm{C}$ for $30 \mathrm{~s}, 30 \mathrm{~s}$ at annealing temperature $\left(57^{\circ} \mathrm{C}-61^{\circ} \mathrm{C}\right.$ ), and extension at $72^{\circ} \mathrm{C}$ for $30 \mathrm{~s}$. PCRs were completed with a final extension at $72^{\circ} \mathrm{C}$ for $2 \mathrm{~min}$. Twenty microliter of each PCR product were fractionated by size in a horizontal gel chamber on a $2 \%$ agarose gel containing $0.2 \mu \mathrm{g} / \mathrm{ml}$ ethidium bromide for $60 \mathrm{~min}$ at 185 V. UV-fluorescence was used to visualize the DNA fragments and images were saved using a BioRad ChemiDoc XRS imaging system (Hercules, CA). Following gel electrophoresis, genotypes were recorded in an Excel spreadsheet as $(1,1)$ for homozygous present, $(0,0)$ for homozygous absent, or $(1,0)$ for heterozygous. "Missing data" was coded as $(-9,-9)$. Genotypes for these 172 loci are shown in Additional file 2; Worksheet "Genotypes."

\section{Validation of computational predictions}

Our DNA panel for locus-specific PCR analyses did not include samples from every WGS individual analyzed. Because our representative $T$. gelada individual differed from that supplying the WGS sample used for Alu ascertainment, we used genotype data from PCR analyses for ten Papio individuals on our DNA panel to estimate the validation rate of the computational predictions (Additional file 3). Based on these results, we implemented an additional filtering step on the data in an attempt to minimize the number of false predictions, while continuing to ensure that our interpretation of the computational results was correct. This filter involved re-analyzing the read files for the dataset of $A l u$ insertions present in T. gelada WGS and imposed a minimum length requirement of $30 \mathrm{bp}$ of unique $5^{\prime}$ flanking sequence adjacent to the predicted $A l u$ insertion for the call to be retained. These post-filtered data were sorted as before for the number of shared $A l u$ insertions between $T$. gelada and any two to twelve Papio individuals. The set of candidate loci determined to be present in both individuals of a single Papio species (as reported previously in Jordan et al. 2018), that were also computationally predicted to be shared with T. gelada, were also subjected to the filtering step and those retained were then screened against the [Panu_2.0] baboon genome to eliminate those shared in the $P$. anubis reference genome.

\section{Alu subfamily analysis}

Papio lineage-specific Alu subfamilies evolved from older $A l u \mathrm{Y}$ subfamilies after the baboon stem lineage diverged from its common ancestor with the rhesus macaque [52]. Identification of Alu subfamilies and the corresponding sequence divergence can provide insight regarding the approximate age of an Alu insertion event $[52,57]$. This study included 24 loci ascertained from the baboon genome assembly [Panu_2.0] and another 16 ascertained from the T. gelada WGS with complete Alu sequence available. PCR data indicated that 15 of the 24 [Panu_2.0] set and 8 of the 16 WGS set met the study criteria of being polymorphic among Papio baboons and shared by $T$. gelada. These 23 polymorphic loci were analyzed for Alu subfamily affiliation. Using the genome coordinates in BED format we uploaded a custom track to the UCSC Genome Browser [54] using the Table Browser function. The complete $A l u$ sequence was obtained in FASTA format. Subfamily identification for these elements was determined using an in-house RepeatMasker library [58] (http://www.repeatmasker.org; last accessed November 2019) developed in Steely et al. (2018) [52].

\section{Results}

\section{Computational Alu detection}

Our split-read methods predicted 27,700 Alu insertions in T. gelada WGS data shared among the 12 Papio individuals but absent from rhesus macaque [Mmul8.0.1] (Additional file 4). Because our objective was to target recently integrated $A l u$ insertions present in T. gelada yet polymorphic within Papio, we eliminated 14,744 (53\%) that were present in all twelve Papio individuals. We retained the remaining 12,956 shared by any of two to eleven of the twelve Papio individuals for further analysis. To determine if any particular Papio species or clade was favored or excluded for shared insertion events with $T$. gelada, we sorted the raw output for the number of shared $A l u$ elements in each bin of 2 to 11 individuals (Table 1). Then we counted the number of times a shared insertion was predicted in each Papio individual (Table 1). For example, when an Alu insertion was predicted to be present in any 5 of the 12 Papio individuals and absent from the other 7, we found 294 instances where one of the five individuals with the insertion was $P$. anubis LIV5. All 12 Papio individuals shared hundreds of $A l u$ insertion polymorphisms with T. gelada in all categories. The average of the two individuals of each species $+/$ - the standard deviation is plotted in Fig. 1. A one-way ANOVA with Bonferroni correction detected significant between-group differences for test bins 2 to 10, but not for bin 11. In bin 2, $P$. hamadryas has more shared insertions with $T$. gelada than do P. anubis, P. papio, or P. cynocephalus, while in bin $6, P$. cynocephalus has more shared insertions than the three northern species (Fig. 1; Additional file 1: Table S1). As a group, the northern and southern clades appear to have similar representation overall except as detected in bins 5 and 6 (of 12) in which the southern clade has significantly more shared insertions, on average, than the northern clade $(P \leq 0.05$; Additional file 1 : Table S1). However, the most consistent statistical finding across all bins was for the two $P$. kindae individuals. 
Table 1 Number of T. gelada Alu insertion polymorphisms shared in Papio individuals

\begin{tabular}{|c|c|c|c|c|c|c|c|c|c|c|c|c|c|}
\hline \multirow[t]{3}{*}{ A. } & \multirow[t]{3}{*}{ B. } & \multicolumn{6}{|c|}{ Northern clade } & \multicolumn{6}{|c|}{ Southern clade } \\
\hline & & \multicolumn{2}{|c|}{ P. anubis } & \multicolumn{2}{|c|}{ P. hamadryas } & \multicolumn{2}{|c|}{ P. papio } & \multicolumn{2}{|c|}{ P. cynocephalus } & \multicolumn{2}{|c|}{ P. ursinus } & \multicolumn{2}{|c|}{ P. kindae } \\
\hline & & LIV5 & L142 & 97124 & 97074 & 28547 & 30388 & 16066 & 16098 & 28697 & 28755 & 34449 & 34474 \\
\hline 2 & 1139 & 112 & 122 & 192 & 179 & 136 & 146 & 127 & 140 & 155 & 111 & 486 & 372 \\
\hline 3 & 989 & 174 & 169 & 210 & 205 & 166 & 184 & 227 & 231 & 249 & 185 & 537 & 430 \\
\hline 4 & 944 & 296 & 248 & 268 & 261 & 206 & 259 & 282 & 297 & 343 & 247 & 567 & 502 \\
\hline 5 & 839 & 294 & 290 & 248 & 280 & 241 & 294 & 342 & 375 & 413 & 310 & 574 & 534 \\
\hline 6 & 938 & 396 & 396 & 360 & 381 & 370 & 396 & 491 & 497 & 531 & 421 & 727 & 662 \\
\hline 7 & 851 & 495 & 466 & 448 & 456 & 395 & 430 & 497 & 480 & 505 & 428 & 702 & 655 \\
\hline 8 & 991 & 626 & 638 & 631 & 645 & 546 & 617 & 623 & 663 & 677 & 584 & 849 & 929 \\
\hline 9 & 1171 & 899 & 865 & 830 & 851 & 811 & 869 & 824 & 862 & 894 & 759 & 1040 & 1035 \\
\hline 10 & 1881 & 1659 & 1635 & 1531 & 1522 & 1442 & 1516 & 1501 & 1563 & 1590 & 1405 & 1732 & 1714 \\
\hline \multirow[t]{2}{*}{11} & 3213 & 2980 & 2971 & 2890 & 2907 & 2966 & 3104 & 2811 & 2884 & 3025 & 2636 & 3079 & 3090 \\
\hline & 12,956 & & & & & & & & & & & & \\
\hline
\end{tabular}

The number of Alu insertion polymorphisms ascertained from T. gelada and not fixed in all 12 Papio individuals was calculated to be 12,956 . The distribution of these when shared between any of 2 to 11 of the 12 Papio individuals (column A, 2 to 11 ) is shown in column B. The sum of the values in column B is 12,956 . The ID for each Papio individual is shown at the top of the twelve adjacent columns, for each of the six Papio species, separated by northern and southern clades. The numbers in each column represent the number of times that the shared insertion with $T$. gelada was predicted in that individual. For example, when an Alu insertion was predicted to be shared in 4 of the 12 individuals and absent from the other 8 , one of the four (column A, row 4 ) was $P$. anubis LIV5 296 times and one of the four was $P$. kindae 34474 (BZ11050) 502 times. All 12 Papio individuals share hundreds of Alu insertion polymorphisms with $T$. gelada in all categories. No Papio individuals are preferentially excluded from having shared insertions with T. gelada. ANOVA detected between-group differences in bins $2-10$, but not bin 11. P. kindae has significantly more shared insertion events with T. gelada than all other five Papio species in bins 2 to 4 and 7 to 8 , while significantly more in all except $P$. ursinus in the remaining bins 5, 6, 9 and 10. See Fig. 1

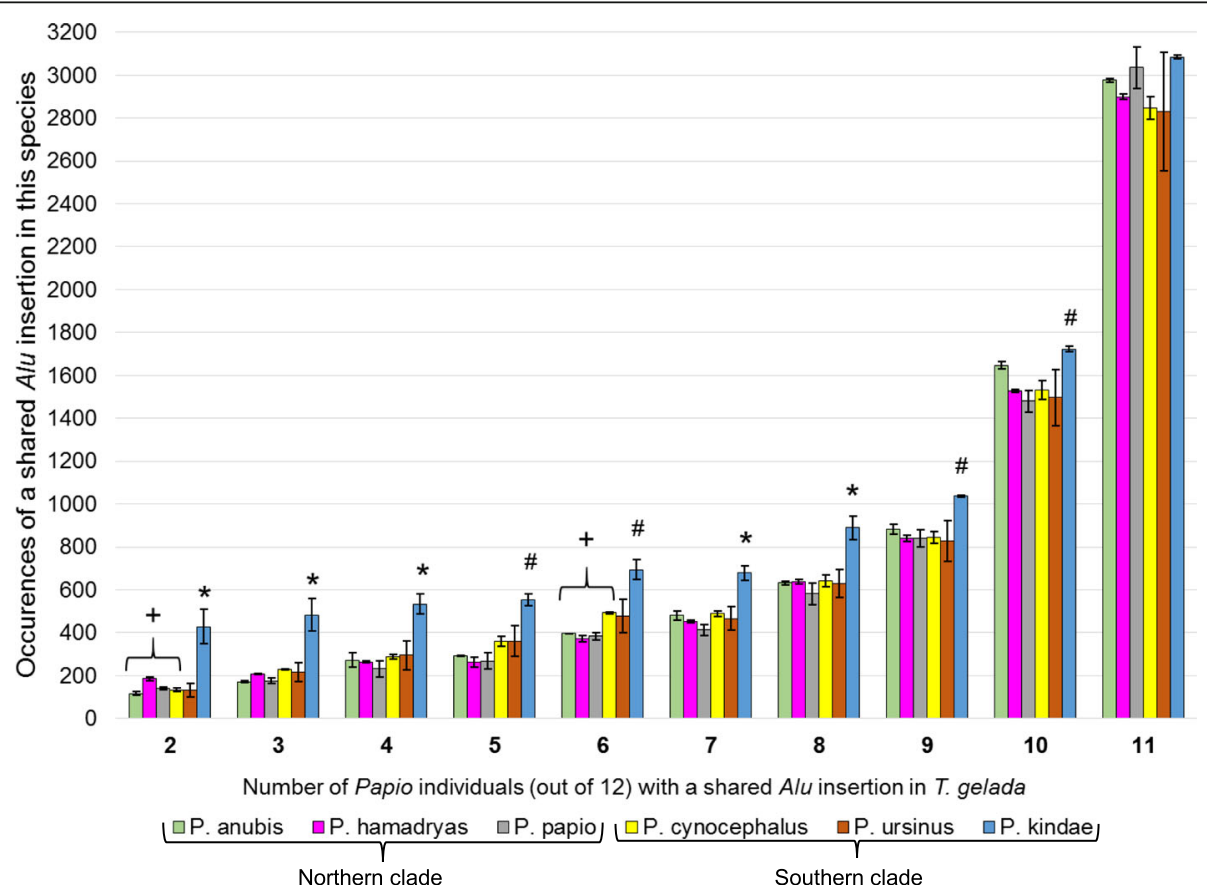

Fig. 1 The number of times a T. gelada-ascertained Alu insertion polymorphism was predicted to be shared in a Papio species when shared in any of 2 to 11 of the 12 Papio individuals. Vertical bars are the average of the two individuals of a given species $+/$ - the standard deviation (error bars). No Papio individuals are preferentially excluded from having shared insertions with $T$. gelada in any category. In bin two, P. hamadryas has significantly more shared insertions than $P$. anubis, $P$. papio, and $P$. cynocephalus (+: $P \leq 0.05)$. In bin six, $P$. cynocephalus has significantly more shared insertions than the three northern species, $P$. anubis, $P$. hamadryas and $P$. papio (+: $P \leq 0.05$ ). Across bins 2 to 10 shared insertions are predicted in $P$. kindae significantly more often than all other five Papio species $\left(^{*}\right)$ or all except $P$. ursinus (\#) $(P \leq 0.05)$ 
P. kindae has significantly more shared Alu insertions with T. gelada than all other five Papio species in bins 2 to 4 and 7 to 8 , while significantly more in all except $P$. ursinus in the remaining bins 5, 6, 9 and 10 (Fig. 1; Additional file 1: Table S1).

These findings prompted us to perform the reciprocal database comparison (B) between the Papio WGS Alu analyses reported in Jordan et al. (2018) [26] and the current WGS Alu database for T. gelada. In that study, $P$. kindae was found to have the most 'species-indicative' Alu insertions with 12,891 elements identified in both $P$. kindae individuals and absent from both the two individuals of all the other five Papio species. We crossreferenced those 12,891 P. kindae Alu loci with the database of 27,700 T. gelada Alu loci to determine if any were shared exclusively between $P$. kindae and T. gelada and identified $236(1.83 \%)$ cases. We performed the same cross-reference analyses for the other five Papio species and found that each of the six Papio species had Alu insertions shared exclusively with T. gelada. P. kindae had significantly more shared insertions than the other five Papio species $(P<0.05)$ (Table 2). The predicted insertion coordinates and sample IDs are listed in Additional file 1, Worksheet "Papio-Theropithecus."

\section{Candidate loci and PCR analyses}

A subset of $150 T$. gelada computationally-derived candidate $A l u$ insertion events were selected for PCR analyses. The oligonucleotide primer design pipeline selected suitable primer pairs using the [Mmul_8.0.1] genome as the mapped reference. After screening these primer pairs against the baboon genome assembly [Panu_2.0], a total of 105 loci were analyzed by PCR for Alu presence / absence within Papio and T. gelada, with 96 generating interpretable results (Additional file 2). PCR based genotypes revealed that 60 of these 96 loci (62\%) met the objective criteria of being polymorphic for insertion presence / absence among Papio baboons and also being shared in a representative T. gelada individual, KB10538 from the San Diego Zoo (DNA was not available for WGS individual 38168). Allele frequency calculations on these 60 loci showed that $P$. hamadryas sample 97124 and $P$. kindae sample 34474 (BZ11050) had the highest counts of shared insertions with 25 and $24 \%$, respectively, while the average across the other Papio samples was 18\% (Additional file 2, Worksheet "allele frequency"). Given that these loci were randomly selected from thousands of candidates, the fact that PCR shows $P$. kindae to have one of the highest frequencies of alleles shared with $T$. gelada supports the computational predictions reported in Table 1.

The second subset of PCR candidates was selected from the dataset of Papio species-indicative elements shared with T. gelada (Table 2). Because we did not have DNA samples from every WGS sample analyzed, including the T. gelada, we randomly selected approximately $10 \%$ of the candidate loci from each Papio species for PCR analysis, with a minimum of five per species. A total of 52 loci from this dataset were analyzed by PCR with 49 generating interpretable results (Additional file 2). PCR results confirmed 26 of these loci contained the candidate Alu insertion in the predicted Papio species and the representative T. gelada individual KB10538 (Additional files 2 and 3). Although 26 of 49 is only about a 53\% confirmation rate from within the candidate loci selected, they provide clear evidence that this particular phenomenon of shared Alu insertion polymorphisms exists in nature, and that each Papio species has multiple Alu insertions also shared in T. gelada but not yet observed in the other five Papio species. An example of this scenario for each of the six Papio species is illustrated with an agarose gel image in Fig. 2.

In addition to the candidate $A l u$ insertion polymorphisms computationally ascertained in this study, subsets $\mathrm{A}$ and $\mathrm{B}$, we also retained 24 loci from previously published studies (12 loci each from $[6,52]$ ) that were ascertained from the olive baboon genome [Panu_2.0] in which PCR experiments indicated the Alu insertion might be shared by Papio and

Table 2 Number of Papio species-indicative Alu insertion polymorphisms shared with Theropithecus gelada

\begin{tabular}{|c|c|c|c|c|c|}
\hline Papio species & $\begin{array}{l}\text { Number of species- } \\
\text { indicative } \\
\text { Alu insertions }\end{array}$ & $\begin{array}{l}\text { Number shared in } \\
\text { T. gelada }\end{array}$ & $\%$ & Z-score & $\begin{array}{l}\text { one-tailed } \\
P \text {-value }\end{array}$ \\
\hline P. anubis & 4645 & 34 & $0.73 \%$ & -0.6821 & 0.2476 \\
\hline P. hamadryas & 8060 & 101 & $1.25 \%$ & 0.1802 & 0.4285 \\
\hline P. papio & 10,873 & 68 & $0.63 \%$ & -0.2445 & 0.4036 \\
\hline P. cynocephalus & 2794 & 26 & $0.93 \%$ & -0.7851 & 0.2162 \\
\hline P. ursinus & 9545 & 57 & $0.60 \%$ & -0.3861 & 0.3498 \\
\hline P. kindae & 12,891 & 236 & $1.83 \%$ & 1.9176 & $0.0276 *$ \\
\hline
\end{tabular}

The number of Alu insertion polymorphisms shared in WGS of Theropithecus gelada that were reported [26] to be exclusive to one Papio species and absent from the other five. $P$. kindae has significantly more such shared elements with $T$. gelada than the other five Papio species $\left({ }^{*} P<0.05\right)$. Z-scores are calculated based on the mean, 87 , and standard deviation equal to 77 
Theropithecus. PCR results using the current DNA panel confirmed that 15 of these 24 met the objective criteria of being polymorphic for insertion presence / absence among Papio baboons while also being shared in our representative $T$. gelada sample. All 172 loci in this study $(96+52+24)$ were confirmed by PCR to be absent in rhesus macaque.

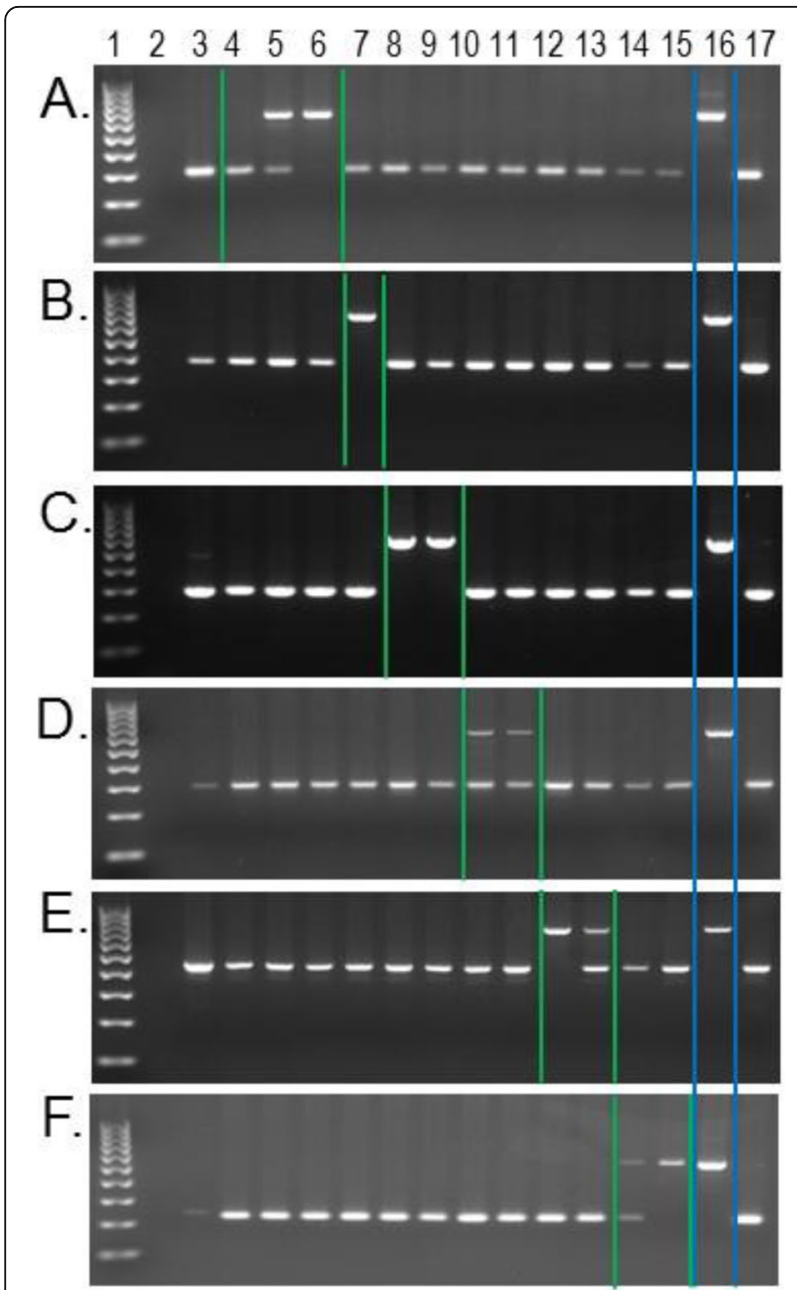

Fig. 2 Papio species-indicative Alu insertion polymorphisms shared in Theropithecus gelada. Lanes: 1- 100 bp ladder, 2- TLE (negative control), 3- Human (HeLa), 4- P. anubis (27861 Panu_2.0 reference individual), 5- $P$. anubis (L142), 6- P. anubis (LIV5), 7- $P$. hamadryas (97124), 8- P. papio (28547), 9- P. papio (30388), 10- P. cynocephalus (16066), 11- P. cynocephalus (16098), 12- P. ursinus (28697), 13- $P$. ursinus (28755), 14- P. kindae (34474; BZ11050), 15- P. kindae (34472; BZ1 1047), 16- T. gelada (KB10538), 17- Macaca mulatta. a olive baboon locus AnuGel_12; b hamadryas locus HamGel_76; c Guinea baboon locus PapioGel_38; d Yellow baboon locus YelGel_11; e chacma baboon locus ChacmaGel_43; $\mathbf{f}$ kinda baboon locus KindaGel_199. Green bars outline the Papio species with the Alu present (upper band); the blue bar outlines the Alu present band in T. gelada

\section{Validation of computational predictions}

We analyzed genotype data for the Papio individuals on our DNA panel to determine the validation rate of the computational predictions (Additional file 3). Of the 96 loci in this dataset that were ascertained from WGS of T. gelada, a total of 206 instances of a filled allele being shared with a Papio individual on our DNA panel were predicted computationally. No PCR amplification occurred in 3 cases, leaving 203 predicted shared cases to analyze. 145 (71\%) of the 203 were confirmed by PCR while 58 (29\%) of the 203 were shown by PCR to be false predictions. Of the 58 false predictions, nearly threequarters $(N=43)$ occurred within 22 loci in which all individuals genotyped as absent for the insertion. A review of all the read files, split-reads and paired-end reads used to make these predictions, provided some clues as to why some predictions were validated by PCR while others were not. PCR-validated predictions typically had multiple supporting reads with at least $40-50 \mathrm{bp}$ of unique $5^{\prime}$ flanking sequence adjacent to the head of the Alu insertion. Predictions not confirmed by PCR tended to have very short ( $\leq 25 \mathrm{bp}$ ) $5^{\prime}$ flanking sequence. This suggests that a lack of flanking sequence to accurately map the split-reads to unique sequence is the likely cause for the majority of the false predictions. Seven $(7.3 \%)$ of the 96 loci were considered 'false negative' in that they were not computationally detected in all 12 Papio individuals (considered polymorphic), but the PCR results indicated the insertion was present in all the Papio individuals on the DNA panel (Additional file 3). This type of error is likely caused by a lack of supporting reads for those individuals such that the insertion is simply not detected, rather than being "predicted absent" by the polyDetect method.

To determine the role of $5^{\prime}$ flanking sequence length on the number of false predictions, we re-analyzed the dataset of 27,700 Alu insertions present in T. gelada WGS that were computationally predicted to be present in any of two to twelve Papio individuals and absent from rhesus macaque [Mmul8.0.1]. We implemented a 'read filter' requiring a minimum of $30 \mathrm{bp}$ of 5 ' flanking sequence adjacent to the predicted Alu (See Methods). These post-filtered data were sorted as before for the number of Alu insertions shared by $T$. gelada and any two to twelve Papio individuals. The post-filtered equivalent of Table 1 is available in Additional file 1: Table S2 and the associated $P$ value for each bin is shown on the same worksheet as Additional file 1: Table S3. The post-filtered equivalent of Fig. 1, using data from Additional file 1: Table S2, is shown in Additional file 1: Figure S1. The number of acceptable candidate loci dropped from 27,700 to 22,875 , with 10,422 (45.6\%) of those determined to be present in all 12 Papio individuals and the remaining 12,453 (54.4\%) were 
determined to be polymorphic among any two to eleven Papio individuals. Although the number of elements in any particular bin shifted somewhat with gains or losses due to the filter requirement, the overall results and interpretation of those results remained the same. All 12 Papio individuals share dozens of Alu insertion polymorphisms with $T$. gelada. Also, as with the original analyses, P. kindae still has significantly more shared Alu insertions with $T$. gelada than any of the other five Papio species in most bins while significantly more in all except $P$. ursinus in bins 4-6. The observable consequences of the filtering step appear to be a reduction in the number of acceptable reads for $P$. anubis sample L142, as compared to the other Papio individuals. Also, the mean values of shared insertions with T. gelada now favor the southern clade over the northern clade more consistently (bins 3-7) than in the previous analyses (bins 5-6). Of the 22 loci containing 43 of the 58 false predictions in the previous analyses, 16 loci and 34 of the 43 false calls were omitted by the filtering step. The number of false predictions was reduced from 58 to 22 and the false prediction rate dropped from 29 to $11 \%$ (Additional file 3). Only one previously validated call was erroneously filtered out. Therefore, the filtered results improved the overall validation rates within this study.

However, the effect of the $30 \mathrm{bp}$ flanking requirement on data reported in Table 2 was more informative. The filter reduced the number of acceptable calls in $P$. anubis sample L142, thus reducing the number found in both P. anubis individuals, LIV5 and L142. The consequence was that some loci were eliminated that had already been PCR validated (i.e. Anu-12 and Anu-6; Additional file 3). Alternatively, the number of predicted P. hamadryas indicative elements included 7 new loci that were not in the original set because they had previous calls in L142 or other Papio individuals that now had been filtered out. Therefore, not only were some reads eliminated, as expected, but this in turn erroneously added loci to each "Papio-indicative" category due to previously called reads in other Papio individuals that were no longer acceptable under the filter conditions. To obtain a value for each Papio species with "high confidence" following the filtering step, we retained only those post-filtered loci also present in the original analyses reported in Table 2, that were also not present in the Panu_2.0 genome. (Additional file 1: Table S4). As before, $P$. kindae still has significantly more shared $A l u$ insertions with $T$. gelada than do the other five Papio species $(* P<0.05)$.

In our attempt to minimize the number of false predictions and improve the validation rate of the polyDetect output in this study, we also inadvertently increased the number of 'false negative' calls dramatically. That is, the absence of a call (no detection in a WGS individual) does not necessarily mean the "predicted absence" of the Alu insertion, only a lack of acceptable mapped reads. Therefore, the filtered results were far less accurate for this metric of the study compared to the first analysis. Also, the errors induced by the filter were more problematic to the overall results of the study than the relatively minor impact of the initial false prediction rate. This highlights the importance of validating methods for data filtering and downstream data processing, and its potential impact on data interpretation. In this case, having a large dataset with overwhelming numbers meant that the overall interpretation was robust to the identified issues.

\section{Papio Alu subfamily distribution}

Of the 172 elements PCR-analyzed in this study, only 23 were suitable for Alu subfamily analysis. They had the complete $A l u$ sequence available from the [Panu_2.0] reference genome and met the study criteria of being polymorphic for insertion presence / absence among Papio baboons while also being shared in T. gelada. These sequences were analyzed for Papio Alu subfamily assignment using an in-house RepeatMasker [58] library developed by Steely et al. (2018) [52]. The RepeatMasker output is available in Additional file 2, Worksheet "RM output". Most of these subfamilies are generally older ancestral subfamilies as shown by their location near the central nodes of the clusters reported in Steely et al. (2018) [52]. The percent divergence from the respective consensus sequences ranged from 0.3 to $3.9 \%$ with the average being $1.8 \%$ ( $\leq 2 \%$ divergence is considered relatively young) $[59,60]$. Of the 23 loci analyzed, 7 were assigned directly to subfamily AluMacYa3, the central node of cluster 1 matching subfamily 0 [52] and the ancestral node originally discovered in Macaca mulatta. Another 11 loci were assigned to Papio Alu subfamilies that derived from AluMacYa3. One locus derived from AluY (3.2\% divergence) while the remaining four loci represented different subfamily clusters but were generally from older rhesus macaque subfamilies such as AluYRa4 (Additional file 2).

\section{Discussion}

The close evolutionary relationship between savanna baboons, genus Papio, and geladas, genus Theropithecus, is well documented $[2,5]$ although recognized as separate genera based on numerous differences in morphology, social behavior and ecology $[4,16,19,21]$. Our finding that about half $(47-54 \%)$ of $A l u$ insertions ascertained from a representative $T$. gelada genome have not reached fixation in the Papio species is unexpected given a Theropithecus / Papio divergence time dating back to 4-5 mya. We also find that each of the six Papio species 
possesses several species-indicative $A l u$ insertions (present in both individuals of that species while absent from all ten individuals from the other five species) that are shared inter-generically with $T$. gelada. This implies a long history of incomplete lineage sorting, admixture and gene flow.

During most of the Plio-Pleistocene, Theropithecus was present throughout much of non-rainforest Africa. Three subgenera are currently recognized: T. (Theropithecus), T. (Simopthecus), and T. (Omopithecus). Of these, $T$. (Theropithecus), including only the extant $T$. gelada, is unknown as a fossil, and may have always been restricted to the Ethiopian highlands. T. (Omopithecus) includes only a single recognized species, $T$. brumpti, confined to the Early Pleistocene of East Africa. The third subgenus, T. (Simopithecus), including T. oswaldi and closely related species, is extensively distributed in time and space, from $\sim 4$ mya to $\sim 100 \mathrm{kya}$, and from southern Africa to Algeria, extending into southern Europe and western Asia [2, 4, 22, 61, 62]. Late populations of $T$. (S.) oswaldi were probably too large in body mass to breed successfully with Papio baboons, but for most of its history, T. (Simopithecus) was comparable in mass to extant baboons.

Some observations of extant baboons and geladas suggest that even after 4 mya of separate evolution, the possibility of gene flow between them is not completely excluded by an intrinsic barrier. A suspected hybrid individual has been observed in a natural gelada-olive baboon overlap zone [63]. In a zoo environment, completely viable first-generation hamadryas baboon $\mathrm{x}$ gelada hybrids of both sexes are reliably reported. While the hybrid males are suspected to be infertile, female hybrids have produced viable offspring by backcrossing to Papio hamadryas [64]. Especially during the earlier phases of their long period of co-existence, Papio $x$ Theropithecus matings (including with $T$. oswaldi) may have allowed ongoing, low-frequency genetic exchange. Our Alu insertion polymorphism data support this hypothesis.

In this study, we also report that $P$. kindae baboons share more Alu insertions with T. gelada than do the other Papio baboons. The reason for this is not well understood and may require further study. Each of the 12 Papio genomes was sequenced to an average read depth of $32.4 x$ coverage with minimum coverage $26.3 x$ [6] and therefore it is unlikely that this finding can be attributed to differences in sequence coverage. An Alubased phylogeny of Papio species placed P. cynocephalus, not $P$. kindae, as most basal within the southern clade [26]. The modern ranges of $P$. kindae and T. gelada are geographically far apart [5, 7]. If they adjoined or overlapped, it might suggest recent hybridization between the two taxa. Moreover, all of the Papio individuals investigated had dozens of shared insertions with T. gel$a d a$, including multiple species-indicative loci. None were preferentially excluded. This suggests that modern geography and habitat are not contributing factors to this finding. Using whole genome comparisons within Papio, the P. kindae genome was found to harbor more species-indicative Alu insertions than the other five species and also found to share more Alu insertions with members of the northern clade that were absent from the other southern clade members [26]. The history of $P$. kindae is reportedly quite unique among baboons. As part of the Baboon Genome Analysis Consortium [6], the best fitting model using coalescent hidden Markov methods indicated that the history of $P$. kindae includes an ancient admixture event involving a lineage related to extant $P$. ursinus from the southern clade (52\% contribution to extant $P$. kindae), with the remaining $48 \%$ contribution to extant $P$. kindae originating from an ancient lineage, possibly extinct, belonging to the northern clade [6]. However, other scenarios may also be possible. If extant P. kindae is the (now geographically restricted) descendent of a geographically widespread ancestral population that exchanged genes with ancestral populations in the Theropithecus lineage and also gave rise to small spin-off populations that expanded one to the north and another to the south, this might also be consistent with the Alu evidence presented in this study.

Our analyses of Alu subfamily distribution are also consistent with a complex evolutionary history for Papio. The ancestral lineages of Asiatic and African papionin monkeys diverged about 8 mya [23]. Alu subfamilies rooted with rhesus macaque, meaning that these subfamilies were active prior to the divergence of Theropithecus / Papio from Macaca, such as AluMacYa3, were shown in this study to have recently integrated progeny elements in Theropithecus / Papio. Many of the $23 \mathrm{Alu}$ insertion polymorphisms analyzed for subfamily assignment had $<2 \%$ divergence from their respective consensus sequences, providing support for their recent integration. The observation that generally older Alu subfamilies have produced the majority of the relatively recent integration events is consistent with the overall estimated divergence timeframe of 4-5 mya. Low Alu sequence variation combined with ongoing persistent levels of insertion polymorphism suggest that the Alu retrotransposition rate among these lineages has been relatively uniform over a long period of time, possibly driven by a lack of reproductive isolation [65].

This study suggests that Papio baboons and Theropithecus have a long history of intertwined evolutionary ancestry that likely includes episodes of intergeneric introgression. A precedent for this among other African primates is available by examining the complex origins of the kipunji, Rungwecebus kipunji. The kipunji is a 
papionin primate discovered in Tanzania in 2003. It was initially assigned to the genus Lophocebus (arboreal mangabey) based on general morphology and arboreal behavior [66] but genetic studies based on mtDNA from a single specimen from Mount Rungwe indicated the new species was more closely related to baboons, genus Papio $[67,68]$. The arboreal mangabey-like phenotype of the kipunji combined with a mtDNA profile similar to a yellow baboon, suggested that Rungwecebus kipunji originated from a hybridization event between a female yellow baboon (Papio cynocephalus) and a Lophocebus male mangabey [69]. It was not until genetic material became available from a kipunji individual from the Ndundulu population about $350 \mathrm{~km}$ away that new evidence suggested that the two kipunji populations likely have different evolutionary histories [70]. The Ndundulu haplotype is considered to be the ancestral or "true" mitochondrial haplotype while the Mount Rungwe population has experienced more recent and perhaps persistent localized introgression from Papio, introducing the observed Papio mtDNA haplotype [71]. The example of the kipunji provides a biological precedent with regard to intergeneric introgression among African primates, similar to our findings between genus Papio and genus Theropithecus.

Following Groves (2001) [3], the tribe Papionini includes macaques (Macaca), mandrills (Mandrillus), terrestrial mangabeys (Cercocebus) and the Highland mangabeys (Rungwecebus kipunji) along with three closely related genera Papio, Theropithecus and Lophocebus [23]. Phylogenetic studies of Papionini have generally separated the genera into two clades, one with Macaca basal to sister taxa Cercocebus and Mandrillus and a second clade consisting of Theropithecus, Papio and Lophocebus, subgenus Papionina [23, 72]. Phylogenetic relationships among the three Papionina genera remain unresolved [23, 73]. Some studies have placed Theropithecus basal to a clade consisting of Papio and Lophocebus [73, 74], while other analyses have placed Theropithecus and Papio as sister taxa, with Lophocebus diverging first [23]. The fact that extensive molecular evidence has yet to resolve this phylogeny suggest possible admixture, reticulation and short internode intervals that facilitate incomplete lineage sorting, and possibly inter-generic hybridization among the lineages.

The increasing availability of vast amounts of WGS data has led to many recent studies being conducted based exclusively on computational analyses, without wet-bench experimental validation to support the genomic comparisons $[75,76]$. Although these reports are informative, this study demonstrates the need to interpret such results with caution. It is important to keep in mind that although "figures don't lie", all forms of data filtering and downstream processing have consequences, some of which are obvious while others are more obscure. Computational data alone may produce interpretable results, but the biological significance of such interpretation should be anchored with experimental evidence when possible. This is especially important when investigating complex phylogenies with an extensive history of admixture and hybridization. Even high quality WGS data from limited sample sizes may not necessarily be representative of the species or genus as a whole, thus molecular validation and adequate sampling are required to support the findings. It is undeniable, however, that the burgeoning availability of WGS data allows greater resolution of complex phylogenies while also recognizing and addressing the impact of confounding factors.

\section{Conclusions}

In this study, we computationally identified over twelve thousand Alu insertions polymorphic in Theropithecus and Papio. Even after incorporating our initial $71 \%$ validation rate and possible $7.3 \%$ false negative error rate, at least $8500 \mathrm{Alu}$ insertions have not reached fixation among the two genera. PCR sequencing based on a small subset of these insertions confirmed over one hundred such cases in support of the computational findings. We also computationally identified over 500 Papio speciesindicative $A l u$ insertions polymorphisms (present in WGS of both individuals of one Papio species while being absent from two samples from each of the other five species) that were determined to be shared in $T$. gelada. PCR evidence confirmed numerous cases of this unexpected phenomenon. All six Papio species have many Alu insertion polymorphisms shared with T. gelada, while $P$. kindae has the largest number. This study suggests that Papio baboons and Theropithecus have a long history of intertwined evolutionary ancestry that likely includes episodes of intergeneric introgression.

\section{Supplementary information}

Supplementary information accompanies this paper at https://doi.org/10. 1186/s13100-019-0187-y.

Additional file 1. An Excel file containing different worksheets for the WGS sample list, Tables S1 - S4, Figure S1 and "Papio-Theropithecus". (XLSX 173kb)

Additional file 2. An Excel file with worksheets for DNA samples, oligonucleotide PCR primers, genomic coordinates, genotype data for the PCR experiments, allele frequency and RepeatMasker output. (XLSX 89kb)

Additional file 3. An Excel file summarizing the $P C R$ validation of computational predictions. (XLSX 45.6kb)

Additional file 4. An Excel file with a list of the 27,700 T. gelada / Papio shared Alu insertions. (XLSX 1.7MB)

\section{Abbreviations}

bp: Base pairs; kya: thousand years ago; mya: million years ago; PCR: Polymerase chain reaction; TPRT: Target primed reverse transcription; WGS: Whole genome sequence 


\section{Acknowledgements}

The authors would like to thank all the members of the Batzer Lab and the Baboon Genome Analysis Consortium for all their helpful suggestions. We also thank Drs. Oliver A. Ryder and Leona G. Chemnick at the San Diego Zoo Institute for Conservation Research for providing T. gelada sample KB10538. Author List: The Baboon Genome Analysis Consortium.

Jeffrey Rogers ${ }^{1,2}$, R. Alan Harris ${ }^{1,2}$, Muthuswamy Raveendran', Yue Liu', Shwetha Murali ${ }^{1 *}$,

Tauras P. Vilgalys ${ }^{3}$, Jerilyn A. Walker ${ }^{4}$, Miriam K. Konkel', Vallmer E. Jordan ${ }^{4}$, Cody J. Steely', Thomas O. Beckstrom 4 , Gregg W.C. Thomas ${ }^{5}$, Kymberleigh A. Pagel $^{6}$, Vikas Pejaver ${ }^{6}$, Claudia R. Catacchio ${ }^{7}$, Nicoletta Archidiacono ${ }^{7}$, Mario Ventura ${ }^{7}$, Alessia Marra-Campanale, Antonio Palazzo ${ }^{7}$, Oronzo Capozzi ${ }^{7}$, Archana Raja ${ }^{8}$, John Huddleston ${ }^{8}$, Veronica Searles Quick, Anis KarimpourFard $^{9}$, Dominik Schrempf ${ }^{10}$, Marc de Manuel Montero ${ }^{11}$, Konstantinos Billis ${ }^{12}$, Fergal J. Martin ${ }^{12}$, Matthieu Muffato ${ }^{12}$, Georgios Athanasiadis ${ }^{13}$, Christina Bergey $^{14}$, Andrew Burrell ${ }^{15}$, Jade Cheng ${ }^{13}$, Laura Cox ${ }^{16}$, James Else ${ }^{17}$, Yi Han ${ }^{1}$, Gisela H. Kopp ${ }^{18,19}$, Maximilian Kothe ${ }^{20}$, Kalle Leppälä̈ ${ }^{13}$, Angela Noll ${ }^{19}$, Jera Pecotte $^{21}$, Lenore Pipes ${ }^{22}$, Karen Rice ${ }^{21}$, Christopher E. Mason ${ }^{22}$, Todd Disotell $^{15}$, Jane Phillips-Conroy ${ }^{23}$, Lutz Walter ${ }^{20}$, Kasper Munch ${ }^{13}$, Thomas Mailund $^{13}$, Mikkel Schierup ${ }^{13}$, Carolin Kosiol ${ }^{10}$, Tomas Vinar ${ }^{24}$, James M. Sikela ${ }^{9}$, Dietmar Zinner ${ }^{18}$, Christian Roos ${ }^{19}$, Clifford J. Jolly ${ }^{15}$, Predrag Radivojac ${ }^{6}$, Roscoe Stanyon ${ }^{25}$, Mariano Rocchi ${ }^{7}$, Evan E. Eichler ${ }^{8,26}$, Bronwen Aken ${ }^{12}$, Matthew W. Hahn ${ }^{5}$, Mark A. Batzer ${ }^{4}$, Tomas Marques-Bonet ${ }^{11}$, Jenny Tung ${ }^{3}$, Donna M. Muzny', Richard A. Gibbs ${ }^{1,2}$, Kim C. Worley ${ }^{1,2}$.

Author Affiliations by Institution.

${ }^{1}$ Human Genome Sequencing Center, Baylor College of Medicine.

${ }^{2}$ Dept. of Molecular and Human Genetics, Baylor College of Medicine.

${ }^{3}$ Dept. of Evolutionary Anthropology, Duke University.

${ }^{4}$ Dept. of Biological Sciences, Louisiana State University.

${ }^{5}$ Dept. of Biology, Indiana University.

${ }^{6}$ Dept. of Computer Science and Informatics, Indiana University.

${ }^{7}$ Dept. of Biology, University of Bari, Bari, Italy.

${ }^{8}$ Dept. of Genome Sciences, Univ. of Washington.

${ }^{9}$ Dept. of Biochemistry and Molecular Genetics, Univ of Colorado Anschutz Medical Campus.

${ }^{10}$ Institute of Population Genetics, University of Veterinary Medicine, Vienna.

${ }^{11}$ ICREA at Institut de Biologia Evolutiva, Universitat Pompeu Fabra, Barcelona.

${ }^{12}$ European Molecular Biology Laboratory, European Bioinformatics Institute, Hinxton.

${ }^{13}$ Bioinformatics Research Center, Aarhus University, Aarhus.

${ }^{14}$ Dept. of Biological Sciences, Norte Dame University.

${ }^{15}$ Dept. of Anthropology, New York University.

${ }^{16}$ Dept. of Genetics, Texas Biomedical Research Institute.

${ }^{17}$ Emory University.

${ }^{18}$ Cognitive Ethology Laboratory, German Primate Center, Gottingen.

${ }^{19}$ Dept. of Biology, Univ. of Konstanz, Konstanz.

${ }^{20}$ Primate Genetics Laboratory, German Primate Center, Gottingen.

${ }^{21}$ Southwest National Primate Research Center, Texas Biomedical Research Institute.

${ }^{22}$ Dept. of Physiology and Biophysics, Weill Cornell Medical College, New

York and HRH Prince Alwaleed Bin Talal Bin Abdulaziz Alsaud Inst. of Computational Biomedicine, Weill Cornell Medicine.

${ }^{23}$ Dept. of Neuroscience, Washington Univ. School of Medicine and Dept. of Anthropology, Washington University.

${ }^{24}$ Faculty of Mathematics, Physics and Informatics, Comenius University, Bratislava.

${ }^{25}$ Dept. of Biology, University of Florence, Florence, Italy.

${ }^{26}$ Howard Hughes Medical Institute.

\section{Authors' contributions}

VEJ, JAW, PG-Q and MAB designed the research and wrote the paper; JAW, VEJ, CJS, PG-Q, CPS, LCR, CER and JMS conducted the experiments and analyzed the results; MKK and TOB performed the Alu repeat analysis of the [Panu_2.0] genome assembly; JMS designed in-house python scripts for data filtering and analyzed the results; VEJ, CJS and TOB performed the computational analysis of the whole genome sequencing data downloaded from the Baylor College of Medicine Human Genome Sequencing Center File Transfer Database; JR contributed the research samples, supervised the work of the Baboon Genome Consortium and edited the manuscript; CJJ revised the manuscript. All authors read and approved the final manuscript.

\section{Funding}

This work was supported by National Institutes of Health Grant R01 GM59290 (M.A.B.).

\section{Availability of data and materials}

The algorithms used in this study are available on GitHub (https://github. com/papioPhlo/polyDetect). The Additional Information files are available on the online version of this paper and through the Batzer Lab website under publications, https://biosci-batzerlab.biology.lsu.edu/. Additional file 1 is an Excel file containing a WGS sample list, Additional file 1: Tables S1-S4, Additional file 1: Figure S1 and worksheet "Papio-Theropithecus." Additional file 2 is an Excel file with worksheets for DNA samples, oligonucleotide PCR primers, genomic coordinates, genotype data for the PCR experiments, allele frequency and RepeatMasker output. Additional file 3 is an Excel file summarizing the PCR validation of computational predictions. Additional file 4 is an Excel file with a list of the 27,700 T. gelada / Papio shared Alu insertions.

\section{Ethics approval and consent to participate}

Not applicable.

\section{Consent for publication}

Not applicable.

\section{Competing interests}

The authors declare that they have no competing interests.

\section{Author details}

${ }^{1}$ Department of Biological Sciences, Louisiana State University, 202 Life Sciences Building, Baton Rouge, Louisiana 70803, USA. ${ }^{2}$ Human Genome Sequencing Center, Baylor College of Medicine, Houston, TX 77030, USA. ${ }^{3}$ Department of Molecular and Human Genetics, Baylor College of Medicine, Houston, TX 77030, USA. ${ }^{4}$ Department of Anthropology, New York University, New York, NY 10003, USA. ${ }^{5}$ Department of Genetics \& Biochemistry, Clemson Center for Human Genetics, Clemson, SC 29634, USA.

\section{Received: 10 September 2019 Accepted: 1 November 2019}

\section{Published online: 26 November 2019}

\section{References}

1. Geoffroy S-HI. Description des Mammifères Nouveaux ou Imparfaitement Connus de la Collection du Muséum d'Histoire Naturelle, et Remarques sur la Classification et les Caractères des Mammifères. Arch Mus Hist Nat Paris. 1843;2:485-592.

2. Jablonski NG. Theropithecus: the rise and fall of a primate genus. Cambridge: Cambridge University press, 2005; 2005.

3. Groves C. Primate taxonomy. Washington, DC: Smithsonian Institution Press; 2001.

4. Jolly CJ. Species, subspecies and baboon systematics. In: LBM WHK, editor. Species, Species concepts and Primate Evolution. New York: Plenum Press; 1993. p. 67-107.

5. Zinner D, Groeneveld LF, Keller C, Roos C. Mitochondrial phylogeography of baboons (Papio spp.): indication for introgressive hybridization? BMC Evol Biol. 2009;9:83.

6. Rogers J, Raveendran M, Harris RA, Mailund T, Leppala K, Athanasiadis G, Schierup MH, Cheng J, Munch K, Walker JA, et al. The comparative genomics and complex population history of Papio baboons. Sci Adv. 2019; 5(1):eaau6947.

7. Zinner D, Wertheimer J, Liedigk R, Groeneveld LF, Roos C. Baboon phylogeny as inferred from complete mitochondrial genomes. Am J Phys Anthropol. 2013;150(1):133-40.

8. Jolly CJ, Burrell AS, Phillips-Conroy JE, Bergey C, Rogers J. Kinda baboons (Papio kindae) and grayfoot chacma baboons (P. ursinus griseipes) hybridize in the Kafue river valley, Zambia. Am J Primatol. 2011;73(3):291-303.

9. Newman TK, Jolly CJ, Rogers J. Mitochondrial phylogeny and systematics of baboons (Papio). Am J Phys Anthropol. 2004;124(1):17-27.

10. Phillips-Conroy JE, Jolly CJ, Brett FL. Characteristics of hamadryas-like male baboons living in anubis baboon troops in the awash hybrid zone. Ethiopia Am J Phys Anthropol. 1991;86(3):353-68. 
11. Rogers J, Kidd KK. Nuclear DNA polymorphisms in a wild population of yellow baboons (Papio hamadryas cynocephalus) from Mikumi National Park. Tanzania Am J Phys Anthropol. 1993;90(4):477-86.

12. Swedell L. African papionins: Diversity of social organization and ecologica flexibility. In: AF CJC, MacKinnon KC, Bearder SK, Stumpf RM, editors. Primates in Perspective. 2nd ed. New York: Oxford Univ. Press; 2011.

13. Cheney DL, Seyfarth RM. Baboon Metaphysics: The Evolution of a Social Mind. Chicago: Univ. of Chicago Press; 2008.

14. Kingdon J, Butynski T. Mammals of Africa: Volume 2 Primates. New York: A\&C Black Publ; 2013.

15. Bergman TJ, Kitchen DM. Comparing responses to novel objects in wild baboons (Papio ursinus) and geladas (Theropithecus gelada). Anim Cogn. 2009;12(1):63-73.

16. Dunbar R, Dunbar P. Social dynamics of gelada baboons. Contrib Primatol. 1975;6:1-157.

17. Gippoliti S. Theropithecus gelada distribution and variations related to taxonomy: history, challenges and implications for conservation. Primates; J Primatol. 2010;51(4):291-7.

18. Snyder-Mackler N, Alberts SC, Bergman TJ. The socio-genetics of a complex society: female gelada relatedness patterns mirror association patterns in a multilevel society. Mol Ecol. 2014;23(24):6179-91.

19. Tinsley Johnson E, Snyder-Mackler N, Lu A, Bergman TJ, Beehner JC. Social and ecological drivers of reproductive seasonality in geladas. Behavior Ecol. 2018;29(3):574-88

20. Zinner D, Atickem A, Beehner JC, Bekele A, Bergman TJ, Burke R, Dolotovskaya S, Fashing PJ, Gippoliti S, Knauf S, et al. Phylogeography, mitochondrial DNA diversity, and demographic history of geladas (Theropithecus gelada). PLoS One. 2018;13(8):e0202303.

21. Delson E. Theropithecus fossils from Africa and India and the taxonomy of the genus. In: Theropithecus: the rise and fall of a primate genus. Cambridge: Cambridge University Press; 1993. p. 157-89.

22. Gilbert CC, Frost SR, Pugh KD, Anderson M, Delson E. Evolution of the modern baboon (Papio hamadryas): a reassessment of the African PlioPleistocene record. J Hum Evol. 2018;122:38-69.

23. Liedigk R, Roos C, Brameier M, Zinner D. Mitogenomics of the Old World monkey tribe Papionini. BMC Evol Biol. 2014;14:176.

24. Boissinot S, Alvarez L, Giraldo-Ramirez J, Tollis M. Neutral nuclear variation in baboons (genus Papio) provides insights into their evolutionary and demographic histories. Am J Phys Anthropol. 2014 155(4):621-34

25. Jolly CJ. A proper study for mankind: Analogies from the Papionin monkeys and their implications for human evolution. Am J Phys Anthropol. 2001; Suppl 33:177-204

26. Jordan VE, Walker JA, Beckstrom TO, Steely CJ, McDaniel CL, St Romain CP, Worley KC, Phillips-Conroy J, Jolly CJ, Rogers J, et al. A computational reconstruction of Papio phylogeny using Alu insertion polymorphisms. Mob DNA. 2018:9:13.

27. Steely CJ, Walker JA, Jordan VE, Beckstrom TO, McDaniel CL, St Romain CP, Bennett EC, Robichaux A, Clement BN, Raveendran M, et al. Alu insertion polymorphisms as evidence for population structure in baboons. Genome Biol Evol. 2017;9(9):2418-27.

28. Szmulewicz MN, Andino LM, Reategui EP, Woolley-Barker T, Jolly CJ, Disotell TR, Herrera RJ. An Alu insertion polymorphism in a baboon hybrid zone. Am J Phys Anthropol. 1999;109(1):1-8.

29. Baker JN, Walker JA, Denham MW, Loupe CD 3rd, Batzer MA. Recently integrated Alu insertions in the squirrel monkey (Saimiri) lineage and application for population analyses. Mob DNA. 2018;9:9.

30. Li J, Han K, Xing J, Kim HS, Rogers J, Ryder OA, Disotell T, Yue B, Batzer MA. Phylogeny of the macaques (Cercopithecidae: Macaca) based on Alu elements. Gene. 2009:448(2):242-9.

31. McLain AT, Meyer TJ, Faulk C, Herke SW, Oldenburg JM, Bourgeois MG, Abshire CF, Roos C, Batzer MA. An alu-based phylogeny of lemurs (infraorder: Lemuriformes). PLoS One. 2012;7(8):e44035.

32. Meyer TJ, McLain AT, Oldenburg JM, Faulk C, Bourgeois MG, Conlin EM, Mootnick AR, de Jong PJ, Roos C, Carbone L, et al. An Alu-based phylogeny of gibbons (hylobatidae). Mol Biol Evol. 2012;29(11):3441-50.

33. Perna NT, Batzer MA, Deininger PL, Stoneking M. Alu insertion polymorphism: a new type of marker for human population studies. Hum Biol. 1992;64(5):641-8.

34. Ray DA, Batzer MA. Tracking Alu evolution in New World primates. BMC Evol Biol. 2005;5:51.
35. Ray DA, Xing J, Hedges DJ, Hall MA, Laborde ME, Anders BA, White BR, Stoilova N, Fowlkes JD, Landry KE, et al. Alu insertion loci and platyrrhine primate phylogeny. Mol Phylogenet Evol. 2005;35(1):117-26.

36. Salem AH, Ray DA, Xing J, Callinan PA, Myers JS, Hedges DJ, Garber RK, Witherspoon DJ, Jorde LB, Batzer MA. Alu elements and hominid phylogenetics. Proc Natl Acad Sci U S A. 2003;100(22):12787-91.

37. Stewart C, Kural D, Stromberg MP, Walker JA, Konkel MK, Stutz AM, Urban AE, Grubert F, Lam HY, Lee WP, et al. A comprehensive map of mobile element insertion polymorphisms in humans. PLoS Genet. 2011;7(8): e1002236.

38. Xing J, Wang $H$, Han $K$, Ray DA, Huang CH, Chemnick LG, Stewart CB, Disotell TR, Ryder OA, Batzer MA. A mobile element based phylogeny of Old World monkeys. Mol Phylogenet Evol. 2005:37(3):872-80.

39. Xing J, Wang H, Zhang Y, Ray DA, Tosi AJ, Disotell TR, Batzer MA. A mobile element-based evolutionary history of quenons (tribe Cercopithecini). BMC Biol. 2007;5:5

40. Xing J, Witherspoon DJ, Ray DA, Batzer MA, Jorde LB. Mobile elements and primate evolution. Yearb Phys Anthropol. 2007;50:2-19.

41. Batzer MA, Deininger PL. A human-specific subfamily of Alu sequences. Genomics. 1991;9(3):481-7.

42. Batzer MA, Deininger PL. Alu repeats and human genomic diversity. Nat Rev Genet. 2002;3(5):370-9.

43. Batzer MA, Stoneking M, Alegria-Hartman M, Bazan H, Kass DH, Shaikh TH, Novick GE, loannou PA, Scheer WD, Herrera RJ, et al. African origin of human-specific polymorphic Alu insertions. Proc Natl Acad Sci U S A. 1994; 91(25):12288-92

44. Ray DA, Xing J, Salem AH, Batzer MA. SINEs of a nearly perfect character. Syst Biol. 2006;55(6):928-35.

45. Stoneking M, Fontius JJ, Clifford SL, Soodyall H, Arcot SS, Saha N, Jenkins T, Tahir MA, Deininger PL, Batzer MA. Alu insertion polymorphisms and human evolution: evidence for a larger population size in Africa. Genome Res. 1997;7(11):1061-71.

46. Cordaux R, Batzer MA. The impact of retrotransposons on human genome evolution. Nat Rev Genet. 2009:10(10):691-703.

47. Roy-Engel AM, Batzer MA, Deininger PL. Evolution of Human Retrosequences: Alu. In: Encyclopedia of Life Sciences (ELS); 2008. p. 1-4.

48. Luan DD, Korman MH, Jakubczak JL, Eickbush TH. Reverse transcription of R2Bm RNA is primed by a nick at the chromosomal target site: a mechanism for non-LTR retrotransposition. Cell. 1993;72(4):595-605.

49. Li H. Aligning sequence reads, clone sequences and assembly contigs with BWA-MEM. Genomics. 2013;1303:1-3.

50. Jurka J, Kapitonov W, Pavlicek A, Klonowski P, Kohany O, Walichiewicz J. Repbase update, a database of eukaryotic repetitive elements. Cytogenet Genome Res. 2005;110(1-4):462-7.

51. Langmead B, Salzberg SL. Fast gapped-read alignment with bowtie 2. Nat Methods. 2012;9(4):357-9.

52. Steely CJ, Baker JN, Walker JA, Loupe CD 3rd, Batzer MA. Analysis of lineagespecific Alu subfamilies in the genome of the olive baboon, Papio anubis. Mob DNA. 2018:9:10.

53. Kent WJ. BLAT--the BLAST-like alignment tool. Genome Res. 2002;12(4): 656-64.

54. Kent WJ, Sugnet CW, Furey TS, Roskin KM, Pringle TH, Zahler AM, Haussler D. The human genome browser at UCSC. Genome Res. 2002;12(6):996-1006.

55. Rozen S, Skaletsky H. Primer3 on the WWW for general users and for biologist programmers. Methods Mol Biol. 2000:132:365-86.

56. Untergasser A, Cutcutache I, Koressaar T, Ye J, Faircloth BC, Remm M, Rozen SG. Primer3--new capabilities and interfaces. Nucleic Acids Res. 2012;40(15):e115.

57. Konkel MK, Walker JA, Batzer MA. LINEs and SINEs of primate evolution. Evol Anthropol. 2010;19:236-49.

58. RepeatMasker Open-3.0. [http://www.repeatmasker.org]. Accessed Nov 2019.

59. Bennett EA, Keller H, Mills RE, Schmidt S, Moran JV, Weichenrieder O, Devine SE. Active Alu retrotransposons in the human genome. Genome Res. 2008; 18(12):1875-83.

60. Konkel MK, Walker JA, Hotard AB, Ranck MC, Fontenot CC, Storer J, Stewart C, Marth GT, Batzer MA. Sequence analysis and characterization of active human Alu subfamilies based on the 1000 genomes pilot project. Genome Biol Evol. 2015:7(9):2608-22.

61. Delson E, Thomas H, Spassov N. Fossil Old World monkeys (Primates, Cercopithecidae) from the Pliocene of Dorkovo. Bulgaria Geodiversitas. 2005;27(1):159-66 
62. Jolly CJ. The classification and natural history of Theropithecus (Simopithecus) baboons of the African Plio-Pleistocene. Bull Brit Mus (Nat Hist) Geology. 1972;22(1):1-123.

63. Dunbar Rl, Dunbar P. On hybridization between Theropithecus gelada and Papio anubis in the wild. J Hum Evol. 1974;3(3):187-92.

64. Jolly CJ, Woolley-Barker T, Beyene S, Disotell TR, Phillips-Conroy JE Intergeneric hybrid baboons. Int J Primatol. 1997;18(4):597-627.

65. Hedges DJ, Cordaux R, Xing J, Witherspoon DJ, Rogers AR, Jorde LB, Batzer MA. Modeling the amplification dynamics of human Alu retrotransposons. PLoS Comput Biol. 2005;1(4):e44.

66. Jones T, Ehardt CL, Butynski TM, Davenport TR, Mpunga NE, Machaga SJ, De Luca DW. The highland mangabey Lophocebus kipunji: a new species of African monkey. Science. 2005;308(5725):1161-4.

67. Davenport TR, Stanley WT, Sargis EJ, De Luca DW, Mpunga NE, Machaga SJ, Olson LE. A new genus of African monkey, Rungwecebus: morphology, ecology, and molecular phylogenetics. Science. 2006;312(5778):1378-81.

68. Olson LE, Sargis EJ, Stanley WT, Hildebrandt KB, Davenport TR. Additional molecular evidence strongly supports the distinction between the recently described African primate Rungwecebus kipunji (Cercopithecidae, Papionini) and Lophocebus. Mol Phylogenet Evol. 2008;48(2):789-94.

69. Burrell AS, Jolly CJ, Tosi AJ, Disotell TR. Mitochondrial evidence for the hybrid origin of the kipunji, Rungwecebus kipunji (Primates: Papionini). Mol Phylogenet Evol. 2009;51 (2):340-8.

70. Roberts TE, Davenport TR, Hildebrandt KB, Jones T, Stanley WT, Sargis EJ, Olson LE. The biogeography of introgression in the critically endangered African monkey Rungwecebus kipunji. Biol Lett. 2010;6(2):233-7.

71. Zinner D, Chuma IS, Knauf S, Roos C. Inverted intergeneric introgression between critically endangered kipunjis and yellow baboons in two disjunct populations. Biol Lett. 2018;14(1):20170729.

72. Harris EE, Disotell TR. Nuclear gene trees and the phylogenetic relationships of the mangabeys (Primates: Papionini). Mol Biol Evol. 1998;15(7):892-900.

73. Guevara EE, Steiper ME. Molecular phylogenetic analysis of the Papionina using concatenation and species tree methods. J Hum Evol. 2014;66:18-28.

74. Harris EE. Molecular systematics of the old world monkey tribe papionini: analysis of the total available genetic sequences. J Hum Evol. 2000;38(2): 235-56.

75. Gao B, Wang S, Wang Y, Shen D, Xue S, Chen C, Cui H, Song C. Low diversity, activity, and density of transposable elements in five avian genomes. Funct Integr Genomics. 2017:17(4):427-39.

76. Lammers F, Blumer M, Ruckle C, Nilsson MA. Retrophylogenomics in rorquals indicate large ancestral population sizes and a rapid radiation. Mob DNA. 2019:10:5.

\section{Publisher's Note}

Springer Nature remains neutral with regard to jurisdictional claims in published maps and institutional affiliations.

Ready to submit your research? Choose BMC and benefit from:

- fast, convenient online submission

- thorough peer review by experienced researchers in your field

- rapid publication on acceptance

- support for research data, including large and complex data types

- gold Open Access which fosters wider collaboration and increased citations

- maximum visibility for your research: over $100 \mathrm{M}$ website views per year

At $\mathrm{BMC}$, research is always in progress.

Learn more biomedcentral.com/submissions 